\title{
The simulation of entropic phase transitions
}

\author{
Daan Frenkel \\ FOM Institute for Atomic and Molecular Physics, Kruislaan 407, 1098 SJ Amsterdam, The \\ Netherlands
}

Received 10 November 1993

\begin{abstract}
This paper reviews recent (numerical) progress in the understanding of entropic phase transitions in complex fluids. In particular, I discuss (liquid-)crystal formation and demixing in binary mixtures. In some cases it appears that lessons learnt in the study of complex fluids may have an unexpected relevance for simple fluids.
\end{abstract}

\section{Introduction}

The second law of thermodynamics tells us that, in a closed system at equilibrium, the entropy, $S$, is at a maximum. A spontaneous phase transformation in a closed system can therefore only occur if it results in an increase of the entropy. However, it is more common to consider the equilibrium behaviour of a system that is not isolated, but can exchange energy with its surroundings. In this case, the second law of thermodynamics implies that the system will tend to minimize its Helmholtz free energy $F=E-T S$, where $E$ is the internal energy of the system and $T$ the temperature. Clearly, a system at constant temperature can lower its free energy in two ways: either by increasing the entropy $S$, or by decreasing the internal energy $E$. In order to gain a better understanding of the factors that influence phase transitions, we must look at the statistical mechanical expressions for entropy. The simplest starting point is to use Boltzmann's expression for the entropy of an isolated system of $N$ particles in volume $V$ at an energy $E$,

$$
S=k_{\mathrm{B}} \ln \Omega
$$

where $k_{\mathrm{B}}$, the Boltzmann constant, is simply a constant of proportionality. $\Omega$ is the total number of (quantum) states that is accessible to the system. In the remainder of this paper, I shall choose my units such that $k_{\mathrm{B}}=1$. The usual interpretation of (1) is that $\Omega$, the number of accessible states of a system, is a measure for the 'disorder' in that system. The larger the disorder, the larger the entropy. This interpretation of entropy suggests that a phase transition from a disordered to a more ordered phase can only take place if the loss in entropy is compensated by the decrease in internal energy. This statement is completely correct, provided that we use (1) to define the amount of disorder in a system. However, we also have an intuitive idea of order and disorder: crystalline solids are 'ordered', while isotropic liquids are 'disordered'. This suggests that a spontaneous phase transition from the fluid to the crystalline state can only take place if the freezing lowers the internal energy of the system sufficiently to outweigh the loss in entropy: i.e. the ordering transition is 'energy driven'. In many cases, this is precisely what happens. It is, however, dangerous and often simply wrong to assume that the intuitive definition of order is equivalent to that based on 
(1). In fact, the aim of this' paper is precisely to show that many 'ordering' transitions that are usually considered to be energy driven may, in fact, be entropy driven. At the outset, I should stress that the idea of entropy-driven phase transitions is an old one, in particular in the theory of complex fluids. The realization that the same ideas may also apply to simple fluids is, however, of a more recent date.

What classes of phase transitions can be driven by entropy alone? In order to answer this question, we must consider systems in which the internal energy is a function of temperature alone. If a first-order phase transformation takes place at constant temperature, the internal energy must remain the same. Hence the change in Helmholtz free energy is determined exclusively by the change in entropy of the system. In general, it is not obvious how to devise model systems for which the internal energy depends only on temperature. In order for this condition to hold, the partition function $Z$ of the system should factorize into a part that depends only on the temperature, $T$, and a part that depends on the density, $\rho$. For a classical $N$-body system, we can write $Z$ as

$$
Z=\frac{1}{h^{3 N} N !} \int \cdots \int \mathrm{d} p^{N} \mathrm{~d} q^{N} \exp \left[-\beta H\left(p^{N}, q^{N}\right)\right]
$$

where $\beta=\left(k_{\mathrm{B}} T\right)^{-1}$, while $H\left(\boldsymbol{p}^{N}, \boldsymbol{q}^{N}\right)$ denotes the Hamiltonian of the system, expressed as a function of the momenta $p^{N}$ and the coordinates $q^{N}$. For convenience, I have assumed that we are dealing with an atomic system. The Hamiltonian $H$ is the sum of the kinetic energy $K\left(p^{N}\right)$ and the potential energy $U\left(q^{N}\right)$. For classical systems, we can perform the integration over momenta in (2) analytically. This yields a factor in the partition function that depends on $T$ only. The remaining, configurational, part of the partition function is

$$
Q=\frac{1}{N !} \int \cdots \int \mathrm{d} q^{N} \exp \left[-\beta U\left(q^{N}\right) .\right.
$$

In general, $Q$ will be a function of $N, V$ and $T$. We are interested in the case where $Q$ does not depend on $T$. It would seem that this is not possible, because $Q$ depends on $\beta$. However, if we limit our attention to hard-core potentials, i.e. potential energy functions that are (depending on the value of $q^{N}$ ) either 0 or $\infty$, then $Q$ is indeed independent of $\beta$. It is easy to see that, in that case, the average potential energy of the system

$$
\langle U\rangle \equiv-\frac{\partial \ln Q}{\partial \beta}=0 .
$$

The average energy of a hard-core system is therefore simply equal to the average kinetic energy $\langle K\rangle$, which is a function of the temperature only. As the internal energy of a hardcore system is constant at constant temperature, any phase transformation in such a system takes place only because this results in an increase in entropy.

Now that we have defined a class of model systems for which entropy is the only driving force behind spontaneous phase transformations, we wish to find out what kinds of phase such a model system can exhibit.

\section{2. (Liquid) crystals}

In this section, I briefly review what we now know about the effect of entropy on the formation of partially ordered liquids ('liquid crystals') and crystalline solids. The 
earliest example of an entropy-driven ordering transition is described in a classic paper by Onsager [1], on the isotropic-nematic transition in a (three-dimensional) system of thin hard rods. Onsager showed that, on compression, a fluid of thin hard rods of length $L$ and diameter $D$ must undergo a transition from the isotropic fluid phase, where the molecules are translationally and orientationally disordered, to the nematic phase. In the latter phase, the molecules are translationally disordered, but their orientations are, on average, aligned. This transition takes place at a density such that $(N / V) L^{2} D=\mathcal{O}(1)$. Onsager considered the limit $L / D \rightarrow \infty$. In this case, the phase transition of the hard-rod model can be found exactly (see e.g. [2]). At first sight it may seem strange that the hard-rod system can increase its entropy by going from a disordered fluid phase to an orientationally ordered phase. Indeed, due to the orientational ordering of the system, the orientational entropy of the system decreases. However, this loss in entropy is more than offset by the increase in translational entropy of the system: the available space for any one rod increases as the rods become more aligned. In fact, we shall see this mechanism returning time and again in ordering transitions of hard-core systems: the entropy decreases because the density is no longer uniform in orientation or position, but the entropy increases because the free volume per particle is larger in the ordered than in the disordered phase.

The most famous, and for a long time controversial, example of an entropy-driven ordering transition is the freezing transition in a system of hard spheres. This transition had been predicted by Kirkwood in the early fifties [3] on the basis of an approximate theoretical description of the hard-sphere model. As this prediction was quite counter-intuitive and not based on any rigorous theoretical results, it met with wide spread scepticism until Alder and Wainwright [4] and Wood and Jacobson [5] performed numerical simulations of the hard-sphere system that showed direct evidence for this freezing transition. Even then, the acceptance of the idea that freezing could be an entropy driven transition came only slowly [6]. However, by now, the idea that hard spheres undergo a first-order freezing transition is generally accepted, and, although the hard-sphere model was originally devised as an idealized and highly unrealistic model of an atomic fluid, it is now realized that this model provides a good description of certain classes of colloidal systems (see the contribution by Pusey in this issue [7]). In fact, we now know that even more complex crystalline order may occur in hard-sphere alloys $[7,8]$. In particular, a binary mixture of hard spheres with a diameter ratio in the vicinity of 0.58 may spontaneously form a substitutionally ordered alloy with the so-called $A_{13}$ structure. Such crystals have a very large unit cell containing 112 particles.

The next step in this sequence came in the mid-eighties when computer simulations [9] showed that hard-core interactions alone could also explain the formation of more complex liquid crystals. In particular, it was found that a system of hard sphero-cylinders (i.e. cylinders with hemi-spherical caps) can form a smectic liquid crystal, in addition to the isotropic liquid, the nematic phase and the crystalline solid. In the smectic (A) phase, the molecules are orientationally ordered but, in addition, the translational symmetry is broken: the system exhibits a one-dimensional density modulation. Subsequently, it was found that some hard-core models could also exhibit columnar ordering [10]. In the latter case, the molecules assemble in liquid-like stacks, but these stacks order to form a two-dimensional crystal. In summary, hard-core interaction can induce orientational ordering and one-, twoand three-dimensional positional ordering. This is rather surprising because, in particular for the smectic and the columnar phase, it was generally believed that their formation required specific energetic interactions. For more details, the reader is referred to a recent review [11]. 


\section{Binary mixtures}

Phase separation in binary mixtures is the example that is used in many textbooks to illustrate the competition between energy and entropy in a phase transformation. For a mixture at constant total volume $V$, the Helmholtz free energy $F$ should be minimal. As a first approximation, the entropy of mixing of a mixture of two species $\mathrm{A}$ and $\mathrm{B}$, is replaced by the entropy of mixing of an ideal mixture

$$
S_{1 \mathrm{~d}}(X)=-N k_{\mathrm{B}}[X \ln X+(1-X) \ln (1-X)]
$$

where $X$ denotes the mole fraction of one component (say A): $X=N_{\mathrm{A}} /\left(N_{\mathrm{A}}+N_{\mathrm{B}}\right)$. The entropy of mixing given by (5) is a convex function of $X$. As a consequence, $S_{\text {id }}(X)$ will always decrease if phase separation takes place. This implies that phase separation can only take place if the resulting decrease in energy $E$ outweighs the increase in $-T S_{\mathrm{id}}$. In a hard-core mixture, there is no energy change upon mixing. Hence, if (5) were exact, we should never observe phase separation in a hard-core mixture, and, even though (5) is known to be only an approximation, it was commonly thought that a fluid mixture of dissimilar hard spheres would not phase separate. This belief was, at least partly, based on the work of Lebowitz and Rowlinson [12], who studied the phase behaviour of such binary mixtures of dissimilar hard spheres, using a more accurate approximation for the entropy of mixing, based on the Percus-Yevick (PY) integral equation. Lebowitz and Rowlinson found that, at least within that approximation, hard spheres of arbitrary size ratio will mix in all proportions in the fluid phase. The implication of this result was that entropic effects are not enough to cause a miscibility gap in a simple fluid mixture. More recently, however, Biben and Hansen [13] have applied a more sophisticated analytical theory for dense fluid mixtures to the same problem. Unlike the PY approximation, this theory is found to predict that an asymmetric binary hard-sphere mixture should phase separate if the sizes of the two spheres are sufficiently dissimilar (typically, if the size ratio is less than 0.2 ). However, as in the case of [12], this is based on an approximate theory for the hard-sphere mixture. Hence, one may wonder to what extent the result found in [13] depends on the approximations that are used to compute the equation of state of the mixture. Clearly, it would be highly desirable to have a model system of a binary mixture for which the existence of a purely entropic demixing transition can be demonstrated unambiguously. In fact, for the case of a hard-sphere mixture, this question has not yet been resolved. However, recent simulations by Dijkstra and Frenkel [15] show unambiguously that entropic demixing does occur in a somewhat simpler binary hard-core mixture, namely a system of dissimilar hard parallel cubes. For an even simpler decorated lattice model [14], the entropic de-mixing transition can be mapped on the magnetic-ordering transition of the Ising model.

Entropic de-mixing is of particular importance in mixtures of particles with differing shapes, for instance spheres and rods or spheres and platelets. One may even argue that the isotropic-nematic transition in a system of infinitely thin hard rods (the Onsager model [1]) is simply a manifestation of the de-mixing transition in a multi-component mixture of parallel hard rods, where every component corresponds to a different orientation. More interesting is the case of a mixture of thin hard rods and spheres. In the limit of very long rods, the solubility of rods in the hard-sphere fluid and spheres in the hard-needle fluid vanishes. The condition for phase coexistence is then only determined by the equality of the pressures of the sphere fluid and the needle fluid. If we assume that the needles are really infinitely thin, then the pressure of the needle fluid is simply the equation of state of an ideal gas, i.e.

$$
P_{\text {needles }}=\rho_{\text {needles }} k_{\mathrm{B}} T \text {. }
$$


The pressure of the hard-sphere fluid is, of course, not known analytically. However, the Carnahan-Starling equation of state is known to reproduce the available simulation data quite well [16]

$$
P_{\text {spheres }}=\rho_{\text {spheres }} k_{\mathrm{B}} T\left(\frac{1+\eta+\eta^{2}-\eta^{3}}{(1-\eta)^{3}}\right)
$$

where $\eta$ denotes the volume fraction of the spheres. The shape of the binodal (envelope of the two-phase region) in a $P_{\text {spheres }}-\rho_{\text {spheres }}$ diagram is given directly by two branches that meet in the origin. One branch, corresponding to the needle fluid, is simply a vertical line at $\rho=0$. The other branch, corresponding to the hard-sphere fluid, is given by the hard-sphere equation of state. This suggests that it should be possible to determine the equation of state of spherical colloids without ever measuring a pressure, simply by bringing the solution of spherical particles in contact with a solution of thin, fairly rigid polymers. Plotting the density of the needle fluid as a function of the density of the sphere fluid should reproduce the hard-sphere equation of state. For more details, see [17].

\section{Depletion flocculation}

Let us next consider a slightly more complex example of an entropy-driven phase separation in a binary mixture, namely polymer-induced flocculation of colloids. Experimentally, it is well known that the addition of a small amount of free, non-adsorbing polymer to a colloidal suspension induces an effective attraction between the colloidal particles and may even lead to coagulation. This effect has been studied extensively [19] and is well understood, at least qualitatively. As in the example discussed above, the polymer-induced attraction between colloids is an entropic effect: when the colloidal particles are close together, the total number of accessible polymer conformations is larger than when the colloidal particles are far apart. However, although the physical mechanism responsible for polymer-induced coagulation is understood qualitatively, a quantitative description of this phenomenon is difficult. This is so because the polymer-induced attraction between the colloidal particles is non-pairwise additive. Moreover, it depends both on the osmotic pressure of the polymer and on the concentration of the colloid. Yet, in the theoretical description of polymerinduced clustering [20], the effect of the polymer is usually replaced by an effective, density-independent, pairwise additive attraction between the colloidal particles. However, in this case, no analytical evaluation of the (grand-canonical) partition function is possible, even when one considers only the very simplest model, viz. that of a mixture of hard-core colloidal particles with ideal chain molecules with conformations that are restricted to a lattice. In this case, it would clearly be desirable to carry out 'exact' numerical simulations to investigate the phase behaviour. Yet, the computational problems are still formidable. What is required is a numerical scheme that samples the positions of the colloidal particles while averaging over all possible conformations of a large (and fluctuating) number of chain molecules. The 'conventional' Monte Carlo schemes to simulate lattice models of polymer systems [18] would be inefficient for such a calculation.

Fortunately, it is possible to construct a rigorous and efficient Monte Carlo scheme to study this model. Our approach relies on the fact that we can recursively compute the partition function of an ideal (non-self avoiding) chain on a lattice in an arbitrary external potential [21-23]. This is most easily seen by considering a chain of length $\ell-1$ on a lattice. For convenience, we assume that the external potential is either zero or infinite. 
The total number of accessible ideal chain conformations that terminate on lattice site $i$ is denoted by $\omega_{\ell-1}(i)$. The total partition function $\Omega_{\ell-1}$ is equal to $\sum_{i} \omega_{\ell-1}(i)$, where the sum runs over all lattice sites. The total number of chains of length $\ell$ that terminate on site $i$ is clearly equal to the sum of the total number of chains of length $\ell-1$ that terminate on any of the neighbours of $i$, multiplied by the Boltzmann factor associated with site $i$. Using such a recursive scheme, we can compute exactly $\Omega_{\ell}$, the partition function of a single ideal polymer of arbitrary length $\ell$ on a lattice, in an arbitrary external potential. This scheme can be used as a starting point to study self-avoiding polymers $[24,25]$, but for the present purpose we limit ourselves to ideal polymers. Up to this point we have not specified the nature of the 'external' potential. We now assume that this potential is due to the presence of $N$ hard, spherical colloidal particles each of which occupies many lattice sites. The polymer partition function clearly depends on the coordinates $r^{N}$ of the colloidal particles: $\Omega_{\ell}\left(r^{N}\right)$. The configurational part of the partition function of the system of $N$ colloids plus one polymer of length $\ell$ in volume $V$ is then given by

$$
Z(V, N, 1)=\int_{V} \mathrm{~d} r^{N} e^{-U_{\text {bs }}\left(T^{N}\right)}\left(\Omega_{\ell}\left(\mathbf{r}^{N}\right)\right)
$$

where $U_{\mathrm{bs}}\left(r^{N}\right)$ denotes the hard-sphere interaction. Next, we make use of the fact that we are considering ideal polymers. In that case we can immediately write down the corresponding partition function for $N$ colloids and $M$ ideal polymers:

$$
Z(V, N, M)=\int_{V} \mathrm{~d} r^{N} e^{-U_{h s}\left(r^{N}\right)}\left(\Omega_{\ell}\left(r^{N}\right)\right)^{M} / M !
$$

where the factor $1 / M$ ! accounts for the fact that the polymers are indistinguishable. Using (7) it is straightforward to transform to an ensemble where the polymer chemical potential (i.e. the osmotic pressure) is kept fixed. The corresponding grand-canonical partition function is given by

$$
\begin{aligned}
& \Xi(V, N, \mu)= \sum_{M=0}^{\infty} \mathrm{e}^{M \mu_{\mathrm{ch}}} Z(V, N, M) / M !=\int_{V} \mathrm{~d} r^{N} \mathrm{e}^{-U_{\mathrm{bs}}\left(r^{N}\right)} \sum_{M=0}^{\infty} \mathrm{e}^{M \mu_{\mathrm{ch}}}\left(\Omega_{\ell}\left(r^{N}\right)\right)^{M} / M ! \\
&=\int_{V} \mathrm{~d} r^{N} \mathrm{e}^{-U_{\mathrm{hs}}\left(r^{N}\right)} \mathrm{e}^{z \Omega_{\ell}\left(r^{N}\right)} .
\end{aligned}
$$

In the last line of (8), we have introduced the polymer fugacity $z \equiv e^{\mu_{\mathrm{ch}}}$, where $\mu_{\mathrm{ch}}$ denotes the chemical potential of the chain molecules.

The important point to note is that (8) allows us to evaluate the properties of the colloidal particles in osmotic equilibrium with a polymer reservoir. In particular, it shows that we can perform Monte Carlo sampling of the colloidal particles. The polymers only affect $U_{\text {eff }}\left(r^{N}\right)$, the effective interaction between the colloidal particles:

$$
U_{\text {eff }}\left(r^{N}\right) \equiv U_{\text {hs }}\left(r^{N}\right)-z \Omega_{\ell}\left(r^{N}\right) .
$$

$z \Omega_{\ell}\left(r^{N}\right)$ measures the entropic interaction between the colloids due to all possible polymer conformations. This entropic interaction is, in principle, not pairwise additive. In fact, it is shown in [22] that, for all but the shortest chain molecules, this non-additivity of the polymer-induced interaction between the colloids has a pronounced effect on the structure and stability of the mixture. In particular, if the non-additivity of the polymer-induced 
interaction is ignored, the range of the colloidal 'liquid' is seriously underestimated [23]. Both the simulations and the experiments on real colloid-polymer mixtures [7] show that the size of the polymers relative to the colloids is crucial for the nature of the phase diagram. If this size ratio is larger than about $1: 4$, the colloid phase diagram contains a crystal, a liquid and a vapour regime. For smaller polymers, however, the liquid-vapour transition disappears (i.e. becomes metastable) and only the solid-'fluid' transition remains. In fact, the disappearance of a stable liquid phase appears to be a general phenomenon in cases where the range of the (effective) attraction between the particles becomes sufficiently short. The attractive feature of polymer-colloid mixtures is that one can vary the range of the effective colloid-colloid attraction simply by changing the size of the added polymer. In simple atomic or molecular fluids one does not have the same freedom in 'tuning' the intermolecular potential. In fact, for most simple liquids ( $\mathrm{Ar}, \mathrm{Kr}, \mathrm{Xe}, \mathrm{CH}_{4}$ etc) the shape of the intermolecular potential is to a good approximation described by the Lennard-Jones $(12-6)$ potential. Therefore, all these materials obey the same law of corresponding states. In particular, they all have a liquid phase. There is, however, at least one simple molecular species that has a pair potential that is much shorter ranged than the Lennard-Jones $12-6$ potential, viz. $\mathrm{C}_{60}$. In fact, recent simulations by Hagen et al [26] suggest that $\mathrm{C}_{60}$ may not have a stable liquid phase at all.

\section{Acknowledgments}

This work is part of the research program of FOM (Foundation for Fundamental Research on Matter), and is supported by The Netherlands Organization for Scientific Research (NWO). I gratefully acknowledge the contributions of Peter Bolhuis, Maarten Hagen, Germonda Mooij, Evert Jan Meijer and Henk Lekkerkerker.

\section{References}

[1] Onsager L 1949 Proc. NY Acad. Sci. 51627

[2] Kayser R F and Raveche H J 1978 Phys. Rev. A 172067

[3] Kirkwood J E 1951 Phase Transformations in Solids ed R Smoluchowski, J E Mayer and W A Weyl (New York: Wiley) p 67

[4] Alder B J and Wainwright T E 1957 J. Chem. Phys. 271208

[5] Wood W W and Jacobson J D 1957 J. Chem. Phys. 271207

[6] Percus J K (ed) 1963 The Many-Body Problem (New York: Interscience)

[7] Pusey P N 1994 J. Phys.: Condens. Matter 6 A29

[8] Edlridge M D, Madien P A and Frenkel D 1993 Nature 36535

[9] Frenkel D, Lekkerkerker H N W and Stroobants A 1988 Nature 332822

[10] Veerman J A C and Frenkel D 1992 Phys. Rev. A 455633

[11] Allen M O, Evans G T, Frenkel D and Mulder B M 1993 Adv. Chem. Phys. 861

[12] Lebowitz J L and Rowlinson J S $1964 \mathrm{~J}$. Chem. Phys. 41133

[13] Biben T and Hansen J P 1991 Phys. Rev. Lett. 662215

[14] Frenkel D and Louis A A 1992 Phys. Rev. Lett. 683363

[15] Dijkstra M and Frenkel D 1994 submitted

[16] Carnahan N F and Stariing K E 1969 J. Chem. Phys. 51635

[17] Bolhuis P and Frenkel D 1994 to be published

[18] Kremer K and Binder K 1988 Comput. Phys. Rep. 7259

[19] For a review and other references see Napper D H 1983 Polymeric Stabilazation of Colloidal Dispersions (London: Academic) ch 6

[20] See, for example, Vincent B, Edwards J, Emmett S and Groot R 1988 Colloids Surf. 31267 
[21] Frenkel D 1990 J. Phys.: Condens. Matter 2 SA265

[22] Meijer E J and Frenkel D 1991 Phys. Rev. Lett. 671110

[23] Meijer E J and Frenkel D 1993 Tenside; Surfactants-Detergents 30269

[24] Mooij G C A M and Frenkel D 1991 Mol. Phys. 7441

[25] Frenkel D 1991 Physica A 17654

[26] Hagen M H J, Meijer E J, Mooij G C A M, Frenkel D and Lekkerkerker H N W 1993 Nature 365425 